To be published in the Proceedings of the 1994

International Hypervelocity Impact Symposium

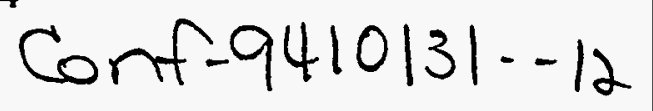

\title{
SWORDS TO PLOWSHARES: SHOCK WAVE APPLICATIONS TO ADVANCED LITHOGRAPHY
}

\author{
Timothy G. Trucano, Dennis E. Grady, Glenn D. Kubiak, \\ Marlin E. Kipp, Richard E. Olson, and Archie Farnsworth \\ Sandia National Laboratories, Albuquerque, NM 87185, U.S.A.
}

\begin{abstract}
Summary-Extreme UltraViolet Lithography (EUVL) seeks to apply radiation in a wavelength region centered near $13 \mathrm{~nm}$ to produce microcircuits having feature sizes 0.1 micron or less. A critical requirement for the commercial application of this technology is the development of an economical, compact source of this radiation which is suitable for lithographic applications. A good candidate is a laser-plasma source, which is generated by the interaction of an intermediate intensity laser pulse (up to $10^{12} \mathrm{~W} / \mathrm{cm}^{2}$ ) with a metallic target. While such a source has radiative characteristics which satisfy the needs of an EUVL source, the debris generated during the laser-target interaction strikes at the economy of the source. Here, we review the use of concepts and computer modeling, originally developed for hypervelocity impact analysis, to study this problem. .
\end{abstract}

\section{INTRODUCTION}

United States industry is working in a collaborative program with the National Laboratories of the Department of Energy to develop Extreme UltraViolet Lithography (EUVL) for the manufacture of microelectronics with 0.1 micron features by the first decade of the 21 st century [1-2]. EUVL employs radiation with wavelengths centered near $13 \mathrm{~nm}$ to achieve high resolution patterning. One of the important criteria that must be met for commercial feasibility of this technology is to develop an efficient, compact, and clean source of the required radiation. The present work is motivated by this need.

Microelectronics fabricated with 0.1 micron features will result in order-of-magnitude increases in transistor densities, processor clock speeds, and DRAM capacities. Thus, lithographic patteming at 0.1 micron resolution will enable the manufacture of 16 GBit DRAM and GHz clock speeds, with aftendant novel applications possibilities [3-4]. Although there are other candidates for microelectronics manufacture at this resolution [1] EUVL has the potential of being used for several manufacturing generations, down to a minimum feature size of approximately 0.03 microns by the middle of the 21 st century [2]. Clearly, the industry would prefer to employ one technology for several successive device generations, rather than change technologies and incur the associated capital costs every few years.

Sandia National Laboratories and AT\&T Bell Laboratories have an ongoing collaboration to confirm the manufacturing feasibility of EUVL by 1996 [5]. Current EUVL systems employ radiation centered at a wavelength of $13 \mathrm{~nm}$ to project a demagnified image of a mask pattern onto a resist-coated wafer. Due to the highly attenuating nature of all the chemical elements at this wavelength, reflective optical elements must be used in the projection imaging systems instead of refractive ones. Reflective elements are coated with periodic multilayer mirrors to achieve reflectance values of $65-70 \%$. Some of these mirrors will have aspheric figures requiring a fabrication precision of $<1 \mathrm{~nm}$ rms. To simultaneously achieve high resolution and adequate exposure field size, EUVL tools that will be used in manufacturing will print with a reduction ratio of $\sim 5 X$ and employ up to 7-8 reflectors. Thus, to achieve production-worthy wafer throughputs, a premium is placed on mirror reflectivity. Additionally, the source of EUV radiation must achieve high flux, its radiation must be efficiently collected to illuminate the object mask, and the resist technology must be both robust and sensitive.

The proof of principal of EUVL for printing at a resolution of 0.05 microns has been established by DISTRIBUTION OF THIS DOCUMENT IS UNLIMITED 


\section{DISCLAIMER}

This report was prepared as an account of work sponsored by an agency of the United States Government. Neither the United States Government nor any agency thereof, nor any of their employees, make any warranty, express or implied, or assumes any legal liability or responsibility for the accuracy, completeness, or usefulness of any information, apparatus, product, or process disclosed, or represents that its use would not infringe privately owned rights. Reference herein to any specific commercial product, process, or service by trade name, trademark, manufacturer, or otherwise does not necessarily constitute or imply its endorsement, recommendation, or favoring by the United States Government or any agency thereof. The views and opinions of authors expressed herein do not necessarily state or reflect those of the United States Government or any agency thereof. 


\section{DISCLAIMER}

\section{Portions of this document may be illegible in electronic image products. Images are produced from the best available original document.}


AT\&T using a synclirrotron radiation source [6] and by Sandia and AT\&T using a more economically feasible compact laser plasma source [7]. Figure 1 shows a schematic diagram of an existing laboratory EUVL system, based on a laser plasma source. The system achieves a resolution close to its design limit of 0.1 micron, as is shown by the printing of 0.1 micron lines and spaces in the resist, shown in the inset of Fig. 1 [8]. In this system, an ellipsoidal condenser, situated close to the plasma source, collects $13 \mathrm{~nm}$ radiation and then focuses it onto the object mask to provide illumination of the mask pattern. A Schwarzschild objective with two spherical mirrors then projects a 10X-reduced image of the mask onto a resistcoated wafer. All of the carefully figured surfaces of the condenser, mask and objective mirrors are coated with an extremely precise periodic multilayer reflective coating comprised of 44 pairs of Mo and Si layers, each of which is $2.5-4.5 \mathrm{~nm}$ thick. Over time, the generation of unwanted debris by the laser plasma source can destroy this mirror coating on the nearby condenser, decreasing system reliability and increasing system cost.

In all of our laboratory systems, a laser plasma source of $13 \mathrm{~nm}$ radiation is employed. The most recent plasma source is driven by a Nd:YAG3+ laser operating at a wavelength of $1064 \mathrm{~nm}$, an intensity on target of $10^{11}-10^{12} \mathrm{~W} / \mathrm{cm}^{2}$, and a repetition rate of $100 \mathrm{~Hz}$. The target material and geometry have been selected to simultaneously optimize EUV generation while minimizing both the generation of target debris and target cost, as is described in more detail in Refs. [9-10]. As we will discuss in greater detail below, the resulting plasma produces $13 \mathrm{~nm}$ centered radiation having good lithographic characteristics for metals with appropriate atomic number $Z$. The dependence of the radiative source characteristics on the target atomic number $Z$, and ultimate conversion efficiency of the laser pulse energy into EUV radiation, have been the subject of much previous work (see [11-13]).

The radiative quality of laser-plasma EUV sources is not an issue. But, unfortunately, when the plasma is formed by the interaction of the laser pulse with a metal target, the source produces a large amount of vaporized and condensed debris which eventually degrades the reflective quality of the multilayer elements $[7,14,15]$. Certain mitigation schemes are available that effectively remove the plasma and neutral vapor component as a threat to the optical elements. But the condensed debris, in the form of ejected solid and melted metal fragments, continues to be troublesome.

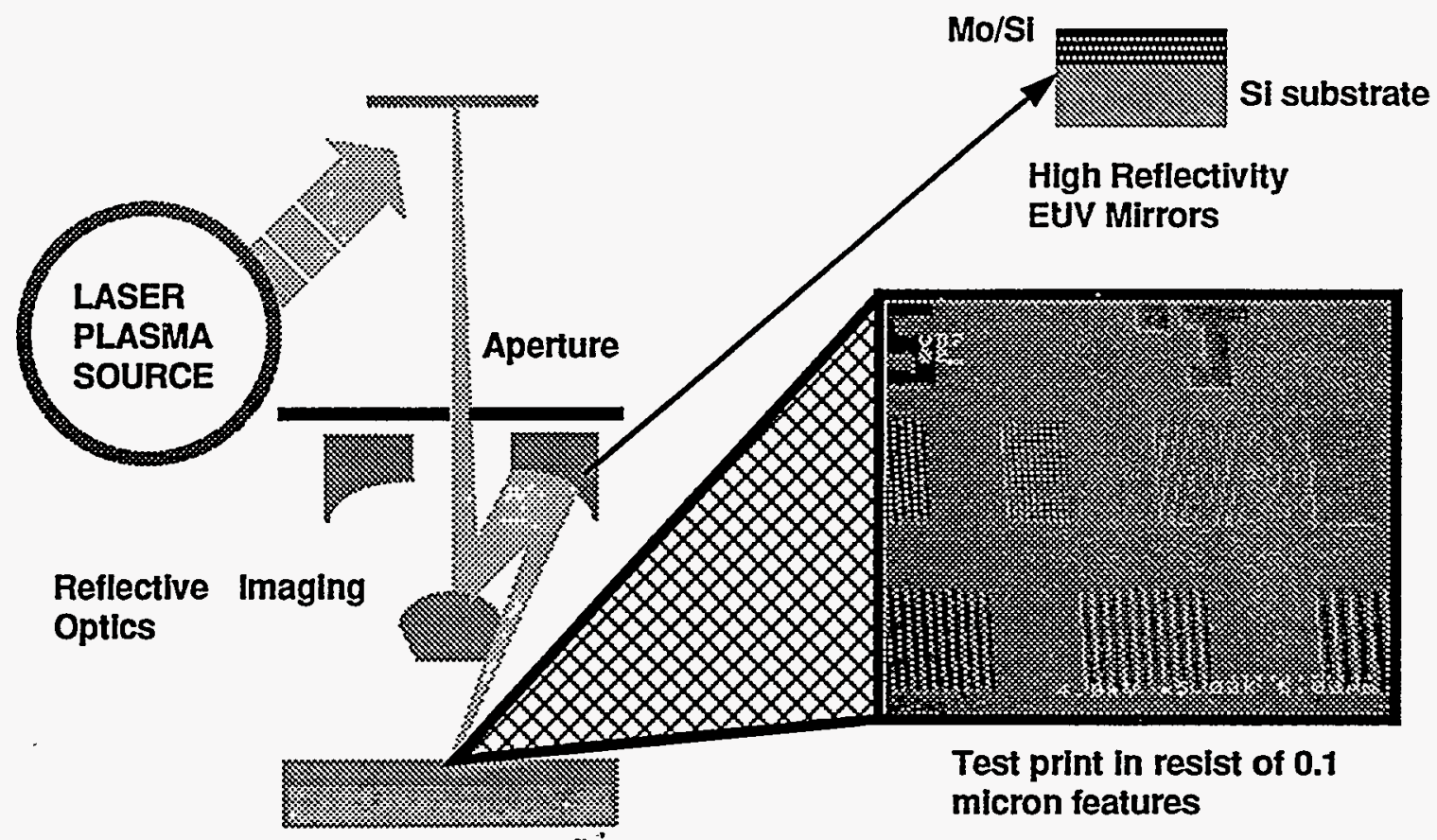

Resist Covered Wafër

Fig. 1. Schematic of a simple $10 \times$ Schwarzschild imaging system achieving its design limiting resolution of $0.1 \mu \mathrm{m}$. The photograph illustrates successful printing of a test pattern consisting of $0.1 \mu \mathrm{m}$ lines and spaces with a laser plasma EUV source at Sandia National Laboratories. 


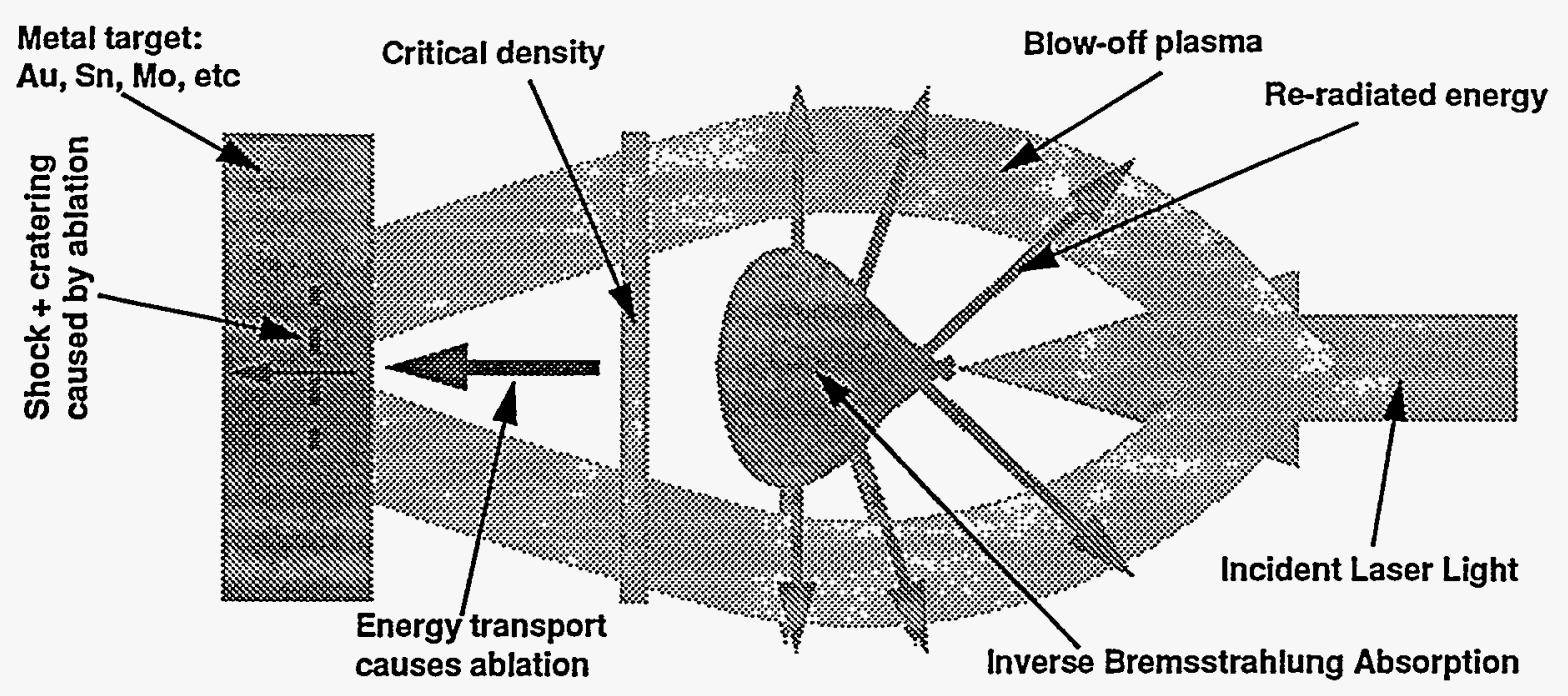

Fig. 2. Schematic of the laser absorption process and subsequent target response. For intensities in the range of interest $\left(10^{11}\right.$ $10^{12} \mathrm{~W} / \mathrm{cm}^{2}$ ), the primary absorption mechanism is classical inverse Bremsstrahlung. All laser absorption takes place at densities which are less than the critical plasma density. Energy is then re-radiated, including a component in the EUV band, and also transported to cold target material via electron thermal conduction. The ablation processes produce ablation pressures that drive shock waves into the cold target and serve to form a crater. The cratering process is an important component in the production of ejecta.

The economic constraints for EUVL are severe [16] and require that the current levels of debris production in the experimental systems be reduced significantly for manufacturing systems. In order to achieve this goal, it is important to better understand the processes of debris production under the laser illumination. It is the purpose of this paper to summarize our work in modeling the debris formation and ejection process for EUVL laser plasma sources. There are three important components of such modeling. First, we model the interaction of single laser pulses with specified metal targets. This modeling allows us to predict radiative behavior of the EUV source over nanosecond time scales and provides an initial condition for further material response calculations. The material response calculations are used to predict crater growth, material fracture, and the initiation of material ejection over microsecond time scales. Finally, debris formation is predicted by dynamic fragmentation theories, using the previously calculated target response as input. Some aspects of this modeling may extend to time scales of milliseconds, given the measured speeds of emerging debris in laboratory situations and the typical distances of optical components from the primary plasma source.

\section{THE LASER-PLASMA SOURCE}

The interaction of laser pulses having intensities in the range $10^{10}-10^{12} \mathrm{~W} / \mathrm{cm}^{2}$ with metal targets is a rather delicate problem for numerical modeling. At these intensities plasma absorption of the light via classical inverse Bremsstrahlung [17] is the dominant absorption mechanism, and anomalous absorption mechanisms are not important, except for long wavelength lasers. When a plasma forms during the pulse absorption, light from the laser is no longer directly absorbed by the cold target. Rather, absorption takes place in a region of density which is smaller than the critical density of the plasma. The energy that drives the subsequent ablation of cold target material then propagates from the plasma absorption region primarily by radiation transport. The absorption region shifts to increasing density (closer to the cold target) as the wavelength of the laser light shortens. Re-radiation of incident laser light from the absorption region, partially as EUV radiation, also occurs. Thus, the dominant energy balance in the pulse absorption consists of ablation and energy coupled to the cold target, kinetic and internal energy in the expanding plasma, and radiative energy emitted from the plasma. A schematic of the absorption in presented in Figure 2.

Because of the relatively low intensity of these incident laser pulses, both plasma which is not highly ionized and neutral target vapor are of importance to the ablation. This is unlike the case for the much higher intensity incident pulses of inertial confinement fusion (ICF), where highly ionized plasmas are dominant. Energy flow during pulse absorption is sensitive to the details of the ionization, and we should be careful in applying models that have been mainly validated for ICF applications. Some discussion of the problems peculiar to modeling absorption of laser pulses at intensities of interest in this study 
may be found in [18]. Since a numerical model of the pulse absorption is used as the driving condition for our subsequent material response calculations, we would like this model to be as accurate as possible.

We have chosen to use the LASNEX [19] 2-D axisymmetric laser inertial confinement fusion (ICF) code to model the laser pulse absorption process, rather than attempt to build a laser absorption model specific to EUVL laser pulses. As mentioned above, we need to be concerned about applying this code to our relatively low intensity laser pulses. However, LASNEX does provide the qualitatively accurate laser absorption models that we need, and detailed models for the radiative behavior of the plasma. The total duration of these calculations is the duration of the laser pulse. The laser pulses are approximately Gaussian, with temporal full-width half-max's (FWHM) of approximately 5 to $34 \mathrm{~ns}$, depending upon the laser. The KrF laser that we discuss the most in this paper has a 34 ns FWHM. LASNEX simulations of this pulse have a total duration of approximately $45 \mathrm{~ns}$.

The predictive power of LASNEX for EUVL applications has been tested by simulating EUVL source characteristics. Significantly accurate predictions of the radiating region have been achieved in modeling EUV emission from laser plasma sources, such as accurate prediction of the trend in conversion efficiency of laser light to EUV as a function of atomic number $Z$. For example, Cerjan [13] has successfully compared spectral data for the interaction of an Nd:YAG laser (1.06 $\mu \mathrm{m}$ light) with tin at intensities on the order of $10^{12} \mathrm{~W} / \mathrm{cm}^{2}$. In the Sandia/AT\&T program, we have had considerable success in simulating the radiative source behavior of a $\mathrm{KrF}$ laser $(0.25 \mu \mathrm{m}$ light) interacting with gold [20-22]. We illustrate the accuracy with which LASNEX can model the radiative characteristics of this particular plasma in Figure 3. There, we show Be-filtered time-integrated $x$-ray pinhole images from an experiment and the corresponding LASNEX prediction (using the post-processing package TDG). The size and shape of the emitting region is quite accurately simulated by LASNEX. For long laser pulses (30-45 ns FWHM) LASNEX calculations of this type also successfully predicted that EUV emission intensity begins to decrease while the laser pulse intensity is still increasing. This is now known to be due to a plasma "shuttering" effect, in which the plasma expands between the detector and the emission region. This type of agreement is strong evidence that we are modeling the hydrodynamics of the plasma expansion accurately.

Most of the work on modeling the laser pulse absorption for EUVL applications has focused on two issues: (1) maximizing the conversion efficiency (CE) of the laser pulse into radiation centered around $13 \mathrm{~nm}$ and (2) tuning of the characteristics of the radiating region. Optimization of the optical system is ultimately sensitive to the temporal and spatial characteristics of the source, and therefore it has been of interest to examine various means of tuning the source characteristics through laser pulse characteristics. There has been considerable modeling success in this endeavor.

The debris problem has not been dealt with directly in previous radiative studies of the source, although it has been recognized that an optimal target from the standpoint of $C E$ is not necessarily optimal for debris production [15]. For example, a metal having an excellent CE in the EUV is tin. This metal is also known to produce more debris by mass per fixed laser pulse than any other metal tested thus far in our program. LASNEX does not have appropriate models for describing solid target response. Therefore, to simulate the response of the target beyond the ablation phase we apply the CTH Eulerian hydrocode [23].

\section{CTH CALCULATIONS OF TARGET RESPONSE}

The process of debris formation under the interaction of the laser pulse is sketched in Figure 4. The ablation of metal from the target results in high velocity plasma ejection from the surface. This creates a recoil momentum in the target. The areal dependence of this recoil momentum is determined by the spatial characteristics of the laser pulse and generally leads to crater development, not unlike a hypervelocity impact crater, as indicated in the figure. That ablative crater formation under laser illuminations of sufficient intensity leads to hypervelocity-like craters has been known since the development of the laser. Important references that discuss laser craters as analogs to hypervelocity impact craters are $[24,25]$. The development of such a crater can lead to ejecta in very much the same manner as in impact craters. It is this mechanism that we choose to examine in the following discussion. Another potentially important source of ejecta is surface ejecta arising during the initial phases of the laser pulse absorption and during the main ablation process. The ablation process is hydrodynamically unstable, and can magnify the effects of surface irregularities originally on the target. Some surface ejecta necessarily contribute to the very early time formation of the plasma [26] and could continue to contribute to debris as pulse absorption continues. We will neglect this process in this paper. 

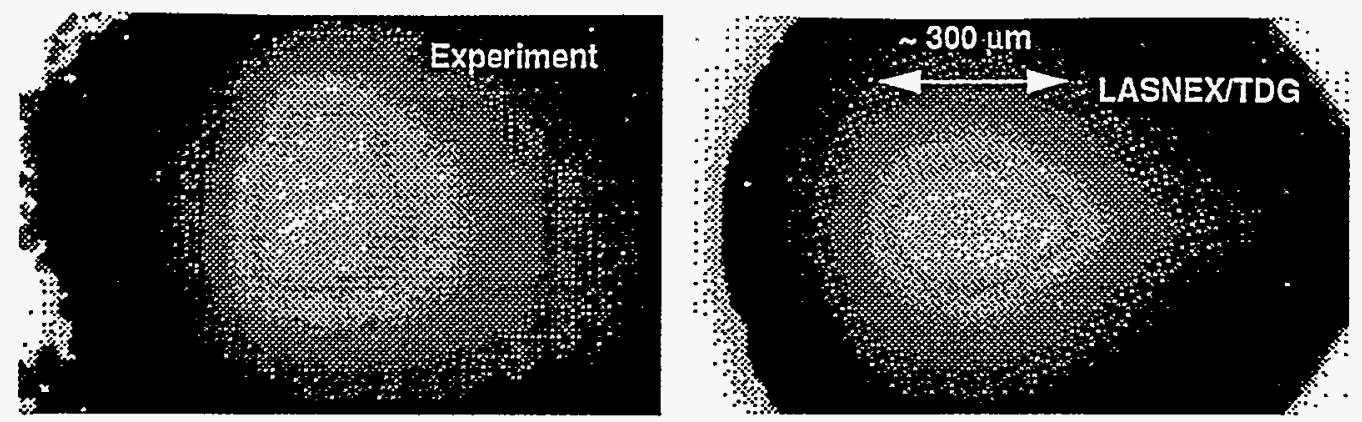

Fig. 3. Comparison of experimental data and LASNEX calculations for a $\mathrm{KrF}$ laser pulse of intensity approximately $7 \times 10^{10} \mathrm{~W} / \mathrm{cm}^{2}$ absorbed in a gold target. We have shown filtered time-integrated $x$-ray pinhole images of the emitting region. Good agreement in both the shape and the size of the emitting region is achieved by the simulation.

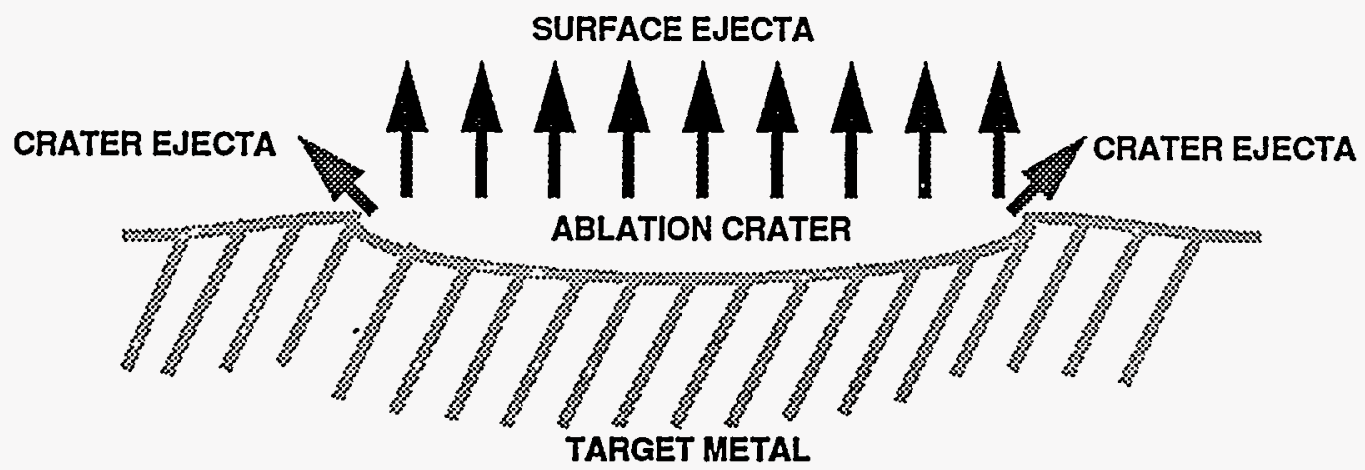

Fig. 4. Schematic of the cratering process due to ablation.

We can make qualitative sense out of the ablation cratering process by developing an analytic model, which allows us to scale the important phenomena. Such a model can then be used to gain perspective on more detailed code calculations, while also providing guidance to the experimental direction for this project. We will suggest such a model in the next section.

Because crater evolution and ejecta formation persist well after the laser pulse ends, solid material response becomes important. Solid response is not modeled accurately by LASNEX and we turn to CTH to continue the numerical modeling of the process beyond the laser interaction regime. CTH models the thermomechanical response of the target to the laser pulse accurately, including phase changes, -material strength, and material fracture. The fracture behavior is a necessary component in our debris model. The final state of the target from the LASNEX calculation of the pulse absorption serves as the initial data for our 2-D axisymmetric CTH calculations. The analytical model discussed below uses most of the fragmentation scheme that we also apply in the CTH calculations. The new information that the hydrocode analysis gives us is a detailed understanding of the temporal and spatial variation of the debris formation process. It offers more information for comparison with the detailed debris data [27,28] than are available experimentally.

While the laser deposition and absorption is a nanosecond time scale event, the target response, crater growth, and debris formation process is typically a microsecond time scale process. We have performed CTH calculations that carry the target response simulation out as long as $10 \mu \mathrm{s}$. Our typical calculations are 1 to $2 \mu \mathrm{s}$, however. Figure 5 illustrates the evolution of a CTH computed ablation crater for a gold target over $1 \mu \mathrm{s}$ after the end of the laser pulse. The surface is slightly perturbed during the pulse absorption, as is visible in Figure 5 at $0.0 \mu \mathrm{s}$. By $0.35 \mu \mathrm{s}$, this perturbation has grown into a full fledged crater. Crater growth has essentially halted by $1.0 \mu \mathrm{s}$, although fragmented material formed around the crater surface has not yet ejected (which will take considerably longer). A residual indicator of the hydrodynamic instability associated with the ablation process is the on-axis jet of less than solid density which is seen at 0.75 and $1.0 \mu \mathrm{s}$.

The details of this jet are not accurately represented by these calculations, and we have not attempted to perform calculations in which such a feature is modeled with greater accuracy. The simulation of surface ejecta is very dependent upon the pulse, the material, the numerical grid in the LASNEX 
calculation, and the'numerical grid in the CTH calculation. Nonetheless, the gross presence of this feature in CTH correctly signifies that unstable surface ejecta are playing a role in the debris formation.

We have examined the effect of the low-density plasma present at the end of the laser pulse on the subsequent development of the target crater and find that crater growth is not significantly affected by this residual plasma. This was established by carefully comparing calculations in which we first keep all of this lower density material and, second, in which we dynamically discard it as the calculation progresses. The crater evolution is identical in both cases. There is often a computational advantage to discarding the low density material because the calculations speed up. When the discarding is used, the crater then looks like Figure 6 (for a slightly different laser pulse).

This type of calculation serves as the basis for analyzing the thermomechanical processes that produce ejecta. Without introducing any additional ideas, we can use such calculations to study the flow of the target material induced by the laser pulse absorption, estimate the amount of material removed from the target (as a bulk quantity), predict experimental observables such as crater size, and map the target material velocity fields. These would provide ejecta velocities and trajectory information that could be compared with experiments. There is considerable data related to the ejecta created by these laser-target interactions, including information about the ejecta mass spectrum. The separate fragments that make up the debris will be smaller than the typical grid resolution used in our CTH calculations, and so additional sub-grid techniques are required to infer ejecta particle information. We have applied such techniques to the analysis of a variety of dynamic fragmentation problems for many years. By applying techniques summarized most recently in [29], we can predict quantities that are comparable to experimental data.

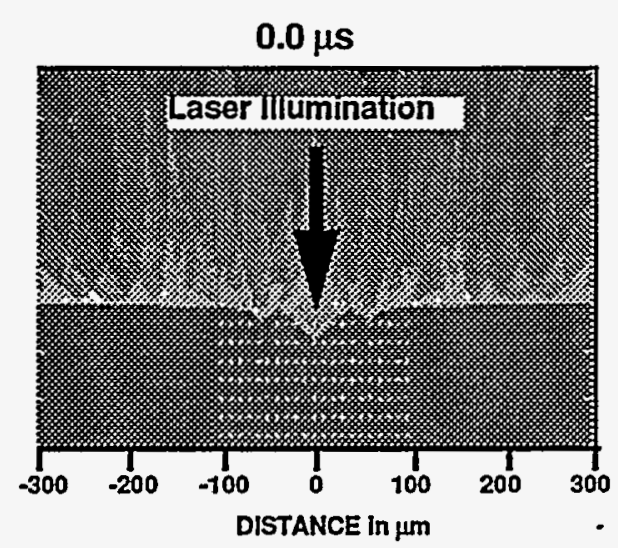

$0.75 \mu \mathrm{s}$

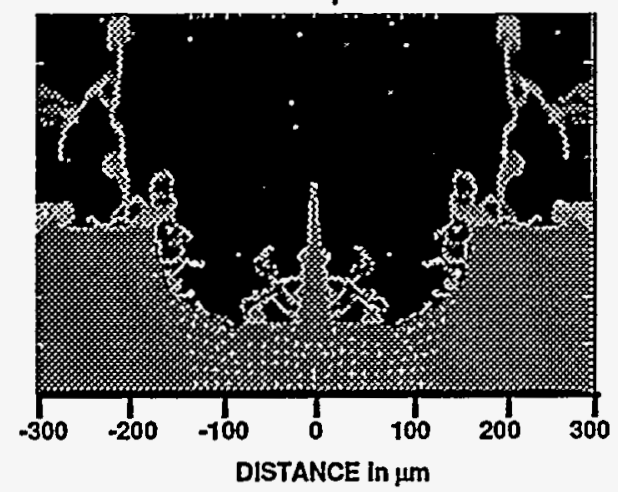

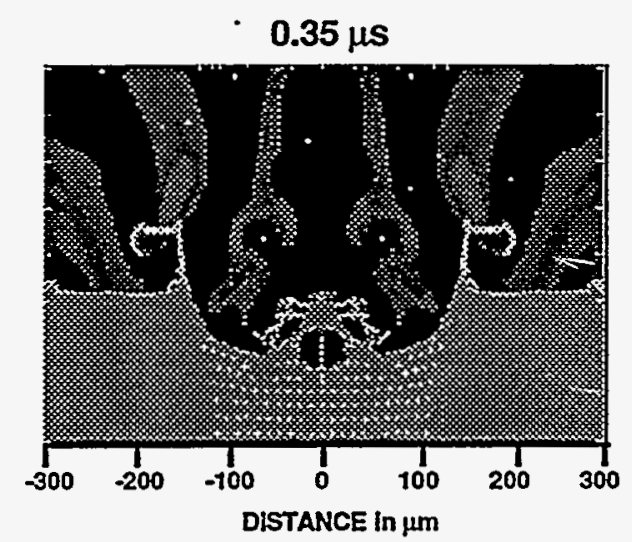

- $1.0 \mu \mathrm{s}$

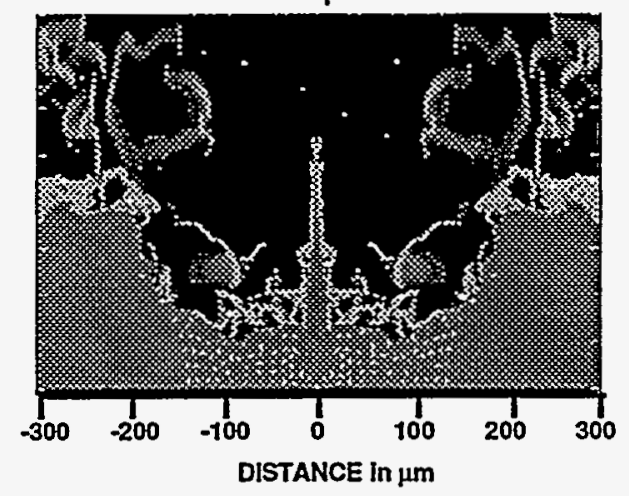

Fig. 5. CTH calculation of the crater evolution in a gold target illuminated by a KrF laser pulse of $34 \mathrm{~ns}$ FWHM duration, and intensity of approximately $10^{11} \mathrm{~W} / \mathrm{cm}^{2}$. Time is measured from the instant of laser shut-off. The hot plasma at the interface of the cold material is visible at $0.0 \mu s$. This plasma rapidly blowsoff and leaves the CTH grid through an outflow boundary condition at the top of the plot. Crater growth occurs due to velocity fields imprinted in the target during the absorption of the laser pulse. Evidence for the hydrodynamic instability of the ablation process is seen in the CTH calculations as a central jet grow/s, having lower than solid density. 


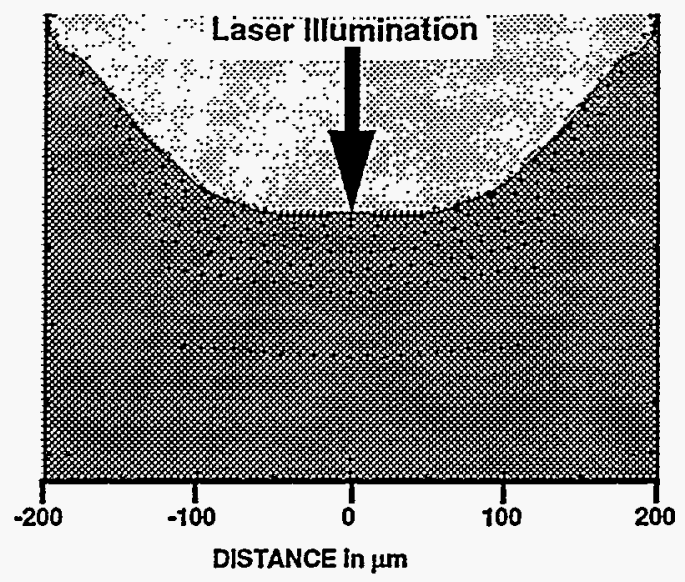

Fig. 6. Residual crater at $1 \mathrm{~ms}$ for a gold target illuminated by a $\mathrm{KrF}$ pulse of approximately $10^{11} \mathrm{~W} / \mathrm{cm}^{2}$ in intensity at $1 \mu s$.

The technique that we apply to estimate detailed fragmentation information is a post-processing technique. The computation of fragmentation information is not intimately coupled to the CTH calculations as they progress. Rather, target response data, as predicted by CTH at selected times, is used to predict fragmentation information. This information yields the observable data that we can compare with experiment, but it is not fed back into the code to modify the subsequent target evolution. This approach has been proven to be accurate in previous applications. The final determination of its accuracy for the present application must occur by comparison with data. We will present the details of how the fragmentation calculation is performed in the section below on the analytic modeling.

Because of the complexity of our strategy for predicting ejecta in EUVL laser-plasma sources, we need to validate this strategy in several stages. For example, the first logical issue to evaluate for accuracy is the pulse absorption. Based on the radiative studies mentioned above, we feel that our LASNEX modeling is quite accurate for the radiative behavior, for both $\mathrm{KrF}$ and $\mathrm{Nd}$ :YAG lasers. However, this alone does not guarantee that the mechanical effects of the pulse on the target are modeled accurately. We should look more closely at the predicted $v s$. actual material response before plunging into fragmentation analysis, given our reservations about the fact that LASNEX has been developed for applications which assume more complete ionization than in the present case.

We know that the crater in Figure 6, as a typical illustration of our calculations, is not an accurate picture of the craters that have been observed in the experiments with $\mathrm{KrF}$ illuminations. Some obvious discrepancies involve the fact that the experimental laser focus is not purely axisymmetric. Thus, the resulting craters are elliptical ( $250 \times 500 \mu \mathrm{m}$ measured edge to edge in a typical case), not circular. This discrepancy is rather small, and is not the source of the main error, which is that the crater in Figure 6 is too deep, and has too large a volume (by an order of magnitude or more). Knowing this, we must ask whether this is due to poorly modeled solid material response during the late stages of the laser pulse absorption, or whether it is more fundamentally related to the modeling of the ablation process. While the radiative behavior in the plasma absorption region seems to be accurate, the ablation region is dominated by electron thermal conduction, which is sensitive to the equation of state and atomic physics modeling of the target material.

We applied an experimental technique using VISAR (Velocity Interferometry System for Any Beflector) velocity interferometry [30] to investigate the mechanical behavior of the target under laser illuminations more directly. A schematic of the experimental technique is presented in Figure 7. The basic data that we acquire from these experiments are time-resolved back-surface velocities of thin targets illuminated by the laser. These velocities are induced by stress waves generated during the ablation of the target by the pulse. As such, they are direct artifacts of the pressure states induced at the illumination side of the target by the laser-induced ablation. The resolution of the VISAR is such that we acquire time-resolved wave profiles of the rear surface velocity. While these experiments are similar to much higher intensity "bum-through" experiments which are common for characterizing the laser-target interaction in ICF work, their time-resolved features are specific to our lower intensity pulses. These experiments will be described in greater detail elsewhere. Here, we will simply state the main conclusions. Typical wave profiles for $\mathrm{KrF}$ pulses of intensities on the order of $5 \times 10^{10} \mathrm{~W} / \mathrm{cm}^{2}$ are shown in Figure 8. Peak axial velocities on the back 
surface are thus less than $150 \mathrm{~m} / \mathrm{s}$ for these three samples, illustrating the relatively low intensity of the original laser pulse. Since no experimental time fiducial was established for these experiments, the curves in Figure 8 were time shifted arbitrarily to all have the same arrival time. The LASNEX simulations of these experiments predicted back surface particle velocities of up to $1.0 \mathrm{~km} / \mathrm{s}$ or more. These are so much greater than the experimental values that we have not bothered to plot the predictions and experiments on the same graph. This discrepancy, coupled with the overestimates of crater volume and other evidence, immediately suggests that LASNEX is incorrectly calculating the ablation pressure for this particular laser interaction. The shorter wavelength of $\mathrm{KrF}$ light $(0.25 \mu \mathrm{m})$ implies a higher critical density, so that it is coupled to the cold target at smaller distances than is Nd:YAG light $(1.05 \mu \mathrm{m})$ and this in principle increases the importance of electron thermal conduction for transporting energy into the cold target. This is one probable source of the ablation pressure error, although experimental uncertainties do not allow a firm conclusion to be reached at this time.

Recently, a more controlled series of VISAR experiments were performed with Nd:YAG laser irradiation at $1064 \mathrm{~nm}$. The spatial quality of the laser pulses for this series was better controlled, and we implemented a technique for establishing an absolute time fiducial. This allowed us to compare predicted vs. measured sample transit times. Among other things, this is a redundant measure of the original ablation pressure that drives the stress waves that we are recording. We also modeled these experiments with LASNEX. A simple comparison with some of the data is summarized in Figure 9. Overall, we are much closer to the peak rear surface velocities for these Nd:YAG pulses than we were for the previous $\mathrm{KrF}$ pulses. The largest difference occurs for the 64 micron gold sample with a pulse energy of $0.9 \mathrm{~J}$ (approximately $10^{12} \mathrm{~W} / \mathrm{cm}^{2}$ intensity).

The conclusions from these experiments is that the ablation pressure computed by LASNEX for the $\mathrm{Nd}$ :YAG pulses in the range $10^{11}$ to $10^{12} \mathrm{~W} / \mathrm{cm}^{2}$ is far more accurate than what we were seeing for $\mathrm{KrF}$ pulses. We confirm the implication of this for crater growth by showing an equivalent calculation of that in Figure 6, but calculated for an Nd:YAG pulse, in Figure 10. There, we see that we have a crater profile which is much closer to those observed in experiments.

More work is required to understand the exact issues that are manifested by the failure of the LASNEX KrF calculations to more accurately predict the ablation pressure. We will discuss the ablation pressure in more detail below and see that scaling laws for ablation pressure, deduced from both ICF experiments and calculations, tend to overpredict the ablation pressure when compared to the data that we have acquired. Thus, we feel that these experiments, and our analysis of them, make an interesting contribution to the literature of the mechanical consequences of coupling high intensity pulses into metal targets.

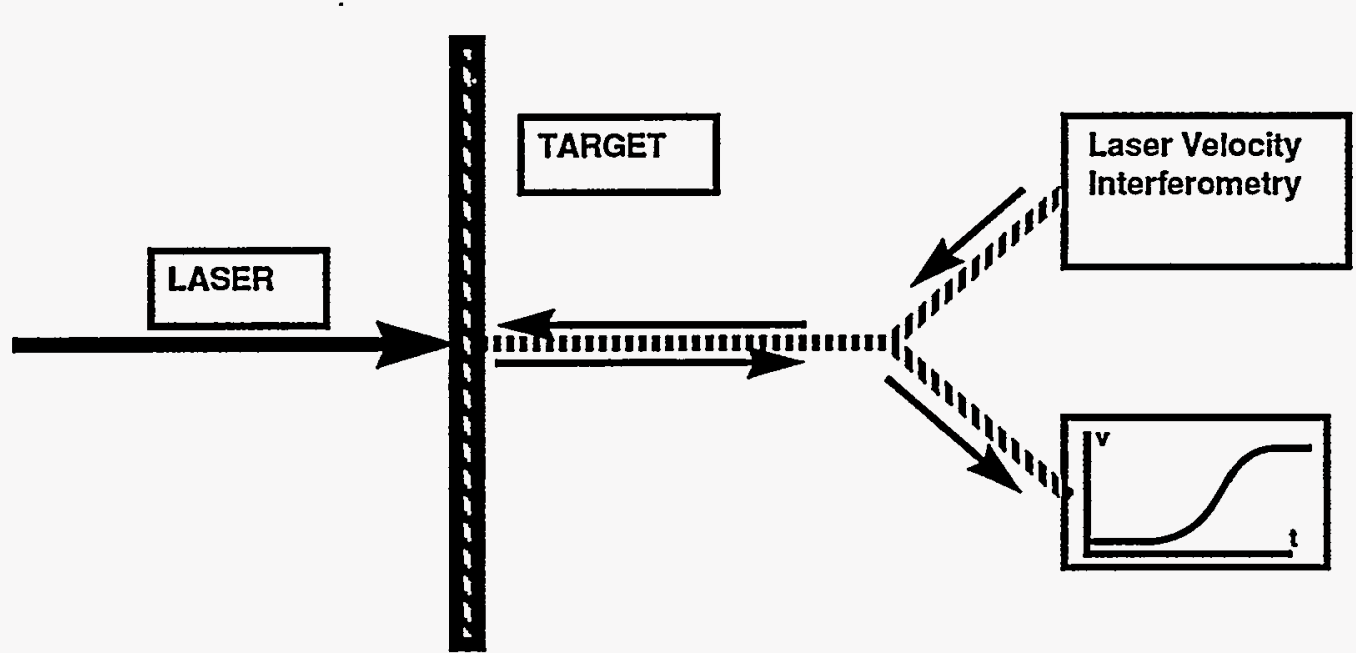

Fig. 7. Schematic of a laser-thin target interaction diagnosed with VISAR velocity interferometry. The laser pulse ablation of the target generates stress waves which propagate along the axis of the beam (assuming normal incidence). When these pulses reflect from the back surface of the target, the induced axial velocity can be measured using this interferometric technique. For large enough spot sizes and thin enough targets, the motion is essentially uniaxial, so that the recorded velocity is an experimental measurement of the axial velocity induced by the waves. 


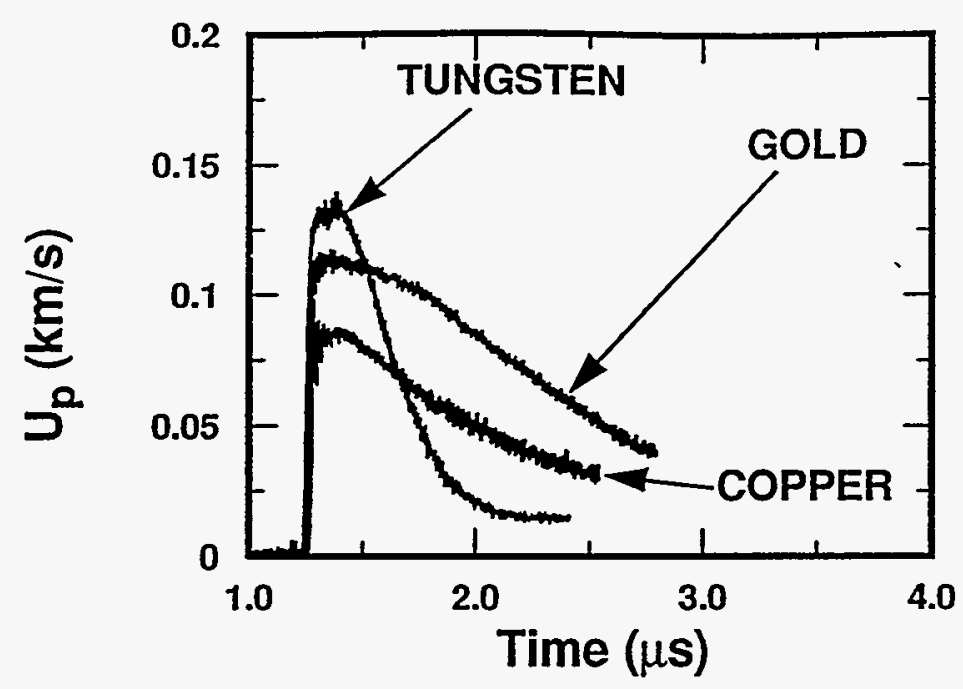

Fig. 8. Wave profiles measured for $25 \mu \mathrm{m}$ thick tungsten, gold, and copper targets. The laser pulse was a $\mathrm{KrF}$ pulse of intensity $\sim 5 \times 10^{10} \mathrm{~W} / \mathrm{cm}^{2} . U_{p}$ is the measured particle velocity at the back surface opposite to the laser pulse.

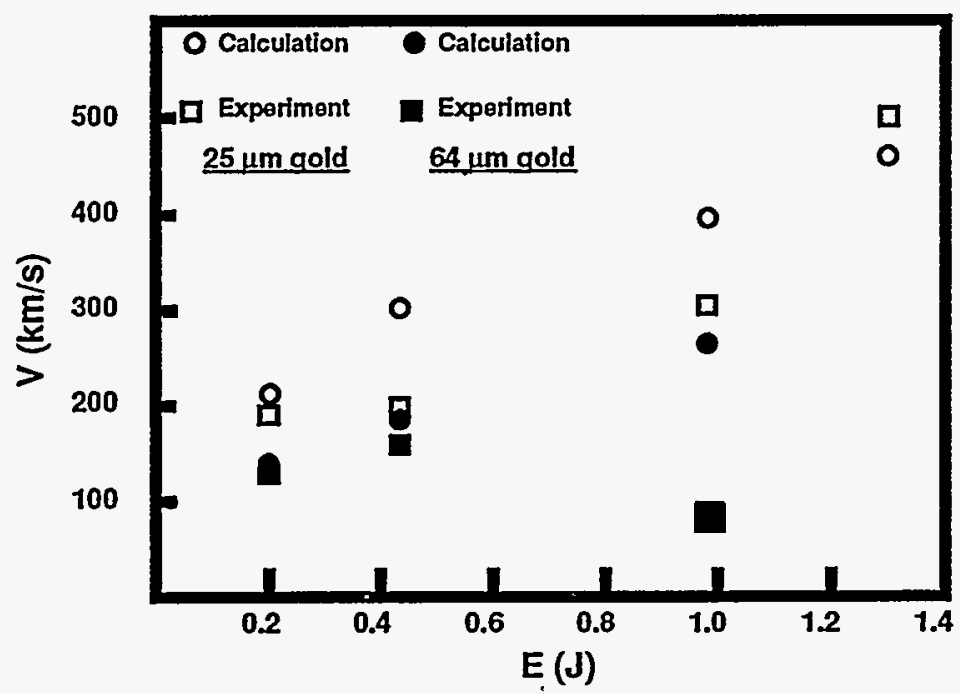

Fig. 9. Comparison of VISAR measurements and LASNEX predictions for Nd:YAG pulses. There are two different sample thicknesses of gold represented in the data

To summarize, the use of the VISAR measurement techniques to quantitatively infer the laser ablation pressures from time resolved experimental data has led to two important pieces of information for computational validation. First, our modeling of the ablation of metal targets by $\mathrm{KrF}$ lasers operating in the intensity range of $10^{10}-10^{11} \mathrm{~W} / \mathrm{cm}^{2}$ over predicts the ablation pressures and, therefore, the predicted crater volumes. Second, our modeling of ablation by Nd:YAG laser pulses having similar intensities is considerably more accurate. These measurements have eliminated uncertainties related to the regime of laser intensities that are important for EUVL, and which are not commonly addressed by the experiments, computations, and scaling studies performed for ICF applications.

\section{AN ANALYTIC MODEL OF CRATER FORMATION}

Simple analytical modeling addresses several needs. First, such a model helps to identify the important physics and parameters in the cratering process that we observe experimentally. This increases our understanding considerably. Second, such a model can guide experimental efforts. Because we are under time and resource limitations in the development of this technology, experimental work is at a premium and must be directed at issues that have the greatest promise of success. Simple modeling can identify the most critical aspects of the parameter space that need to be addressed experimentally. Third, a model can help bound the calculations in much the same way. Time and effort available for extensive computational 
studies are also limited in our program. Beyond this, calculational results must be understood within a reasonable theoretical framework in order to trust them to provide rational guidance for experiments.

We develop a simplified model as follows. First, assume that a radially symmetric pressure pulse is applied to a rigid plastic material, with a given time modulation. We can represent such a pulse by the equation:

$$
P(r, t)=P(r) \cdot F_{0}(t)
$$

We assume that the laser beam is axisymmetric, with a radius $a$. Thus, we intend that $P(r)=0$, if $r \leq 0$ or if $r \geq a$. We also write the total duration of the laser pulse as $\Upsilon$, and then assume that $F_{0}(t)=0$, if $t \leq 0$ or if $t \geq \Upsilon$. For example, as in Figure 11, $F_{0}(t)$ may be Gaussian with a FWHM of $\tau$. Then, $\Upsilon$ is the time cutoff for the pulse. When Equation (1) is inserted in the 1-D momentum balance for the material we get

$$
\rho \frac{\partial u}{\partial t}=-\left(\frac{\partial P}{\partial r}\right)=-F_{0}(t) \cdot \frac{\partial}{\partial r} \bar{P}
$$

where $u$ is the radial material velocity.

We can easily integrate this expression (assuming that $\rho$ is constant in time) to compute $u$ as a function of radius and time:

$$
u(r, t)=-\frac{1}{\rho} \frac{\partial}{\partial r} \bar{P} \cdot \int_{0}^{t} F_{0}(\tilde{t}) d \tilde{t} .
$$

For $F_{0}(t)$ a Gaussian of unit amplitude and FWHM $\tau$, we can then approximate the integral in Equation (3) by $\tau / 2$ for $t \approx r$. Then, we can also write $\int_{0}^{r} F_{0}^{2} d t=\tau / 4$. We then have

$$
u(r, \Upsilon)=-\frac{1}{\rho} \frac{\partial}{\partial r} \bar{P} \cdot \frac{1}{2} \tau
$$

Assuming that the plastic material has a constant yield strength $Y$, we then find that the work done on the material by the assumed pressure is:

$$
W=2 \pi \int_{00}^{a \Upsilon} P(r, t) u(r, t) r d t d r .
$$

By applying the Rankine-Hugoniot conditions behind the shock waves induced by the applied pressure, we can approximate the material velocity by

$$
u \approx \frac{P}{\rho C}
$$

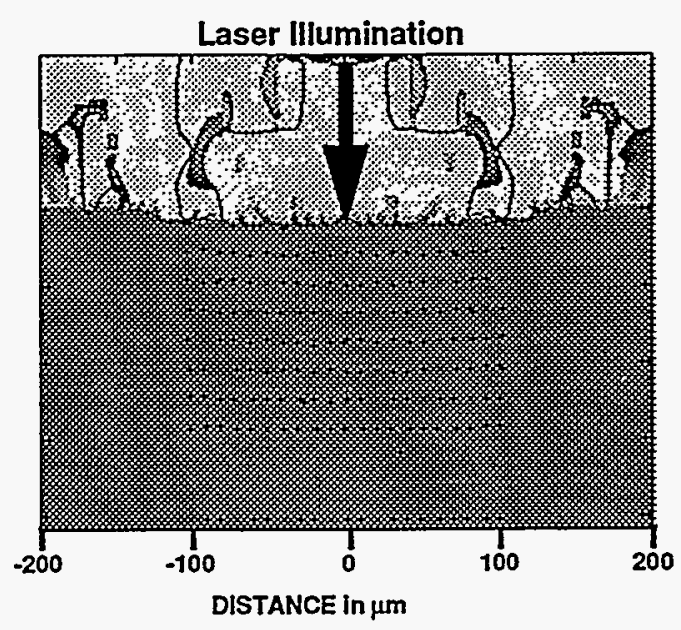

Fig. 10. A CTH calculation of an Nd:YAG pulse $\left(\sim 10^{11} \mathrm{~W} / \mathrm{cm}^{2}\right)$ into a gold target at $10.0 \mu \mathrm{s}$. The crater is far shallower than the $\mathrm{KrF}$ analog in Figure 6, and fairly typical of crater depth vs. diameter aspect ratios observed in EUVL targets. Low density material has not been discarded from this calculation. 
Laser Illumination

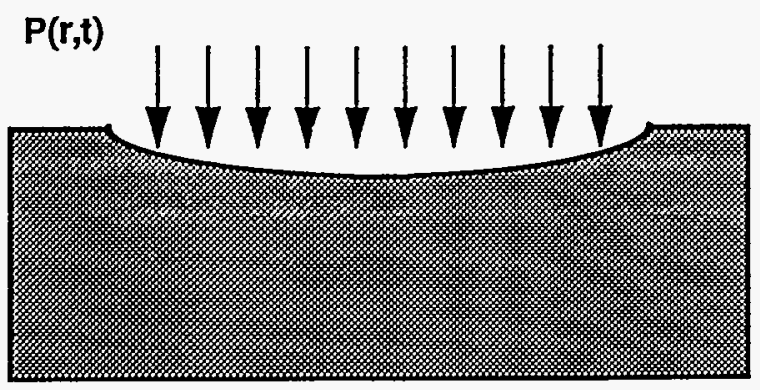

$\overline{\mathrm{P}}(\mathrm{r})$

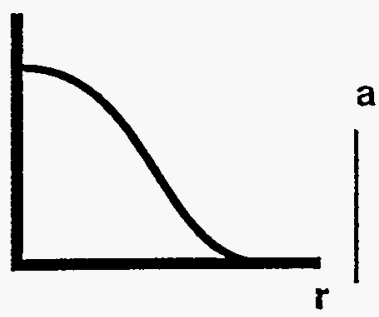

$F_{0}(t)$

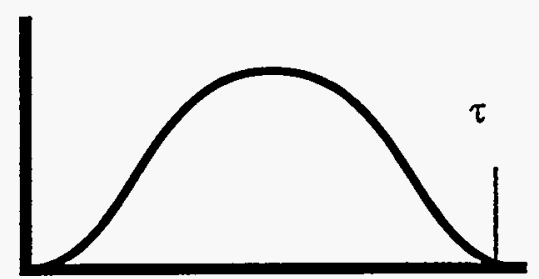

$\mathbf{t}$

Fig. 11. Schematic of the model ablation pressure.

where $C$ is the sound speed in the material behind the shock wave. An alternate expression for the work done in developing a crater of volume $V$ through rigid plastic deformation is $W=V \cdot Y$, assuming a strain of order unity. Setting this equal to Equation (5), we find the following scaling equation for the crater volume under an ablative pressure loading:

$$
V .=\frac{\pi}{Y \rho C} \cdot \frac{\tau}{2} \cdot \int_{0}^{a} P^{2}(r) d r .
$$

Equation (7) is a key formula, in that for a specific radial form of ablation pressure, it suggests important scalings of the resulting crater volume (and thus ejecta) that can be compared with experimental data. We will now discuss specific aspects of the ablation pressure.

Let us assume for simplicity that the radial dependence of the pressure pulse has the following form:

$$
P(r)=P_{\max } \cdot\left[1-\left(\frac{r}{a}\right)^{2}\right] \text {. }
$$

where $P_{\max }$ is the peak amplitude of the ablation pressure (which may be inferred from experiments, computer calculations, or simple scaling laws), and $a$ is the laser focus radius (assuming an axisymmetric spot). When Equation (8) is inserted into Equation (7) we arrive at the following formula for the crater volume:

$$
V=\frac{\pi}{12} \cdot \frac{P_{\max }^{2} \tau a^{2}}{Y \rho C} .
$$

Equation (9) says several important things about the scaling of the crater volume induced by an incident laser pulse. First, and most importantly, it states that the dependence of the crater volume on the ablation pressure is quadratic, making it the most sensitive term of the scaling. The laser ablation pressure is a complex function of laser pulse parameters and target characteristics, as we will show below. Second, Equation (9) shows that the crater volume is inversely proportional to target material strength and shock impedance. This last correlation qualitatively agrees with experimental data.

With the exception of $P_{\max }$, all of the parameters in this formula are easy to evaluate for various target materials and laser pulses, making it rather easy to perform quick assessments of target cratering and fragmentation. Based on the VISAR data that we have acquired for EUVL-type lasers, we expect relatively low ablation pressures, and make the reasonable assumption that

$$
\rho C \approx \rho_{0} C_{0},
$$

with $\rho_{0}, C_{0}$ the ambient values of the target density and sound speed. Since, for any compression, $\rho C>\rho_{0} C_{0}$, this assumption will contribute to an overestimate of the crater volume within the theory:

$$
V=\frac{\pi}{12} \cdot \frac{P_{\max }^{2} \tau a^{2}}{Y \rho_{0} C_{0}}
$$


$P_{\max }$ is the difficult quantity to deal with in Equation (11). In [32], for $\mathrm{KrF}$ laser pulses having intensities on the order of $10^{11} \mathrm{~W} / \mathrm{cm}^{2}$ we inferred from LASNEX calculations that $P_{\max }-200 \mathrm{kbars}$. We now know that this value is not correct from studying the VISAR data. When data such as that presented in Figure 8 are analyzed, the pressures inducing the observed particle velocities are inferred to be on the order of approximately 20 to $50 \mathrm{kbars}$, for intensities on the order of $5 \times 10^{10} \mathrm{~W} / \mathrm{cm}^{2}$. There is mild variation with target material for these low laser intensities, in rough agreement with scaling laws stated in the literature.

In Table 1, we have summarized material parameters and the predicted crater volumes for the specified ablation pressure. These values of $P_{\max }$ marked with an asterisk were inferred from the VISAR experiments, and crudely scaled up to the laser intensities reported in [15]. The others are estimates. We also use the following values in equation (11) to compute the volume data in the table: $\tau=34 \mathrm{~ns}$, $a=75$ microns. The experimental data reported in Table 1 and labeled $V_{E x p}$ was collected in experiments of Kubiak, et al, [15] which were designed to measure the average mass removed per shot for a KrF pulse having the stated characteristics. These particular data were not reported in that paper, however. The column labeled $V_{e}$ is an approximation to the volume of ejected material, as opposed to the volume of the predicted crater $V_{c}^{e}$. It is reasonable to assume that the volume of ejected material is proportional to the volume of the crater in hypervelocity impact events. We assume that this simple proportionality is also true for the laser case. For the sake of argument, we use the Z-scaling theory discussed in Melosh [33]. Z-scaling requires the assumption that debris is ejected at a single angle. For argument's sake, we assume that debris is ejected at a 45 degree angle, leading to the following proportionality:

$$
V_{e}=\frac{1}{4} V_{c}
$$

We have also plotted the data from Table 1 in Figure 12. There, we have normalized the experimental and calculated ejecta volumes separately by the volume of ejecta for tungsten. The trend, clearly reproduced by the model, is that ejecta volume decreases as the yield strength of the target increases. While the magnitudes of the normalized ejecta volumes for the model are quantitatively different than the experimental data, the qualitative agreement with target material variation is very interesting. One slight difference between the model and the experiment is that the model predicts that tantalum produces slightly less ejecta volume than iron, while the experiments show the opposite. Given the wholesale assumptions that entered the model, this deviation is too small to be concerned about. We note that the agreement of the normalized model data with the normalized experimental data in Figure 12 generally improves as the yield strength of the target material increases.

For the pressures listed in Table 1, we tested the approximation Equation (10). We find that the largest difference is for tin, where the predicted ejecta volume decreases by $18 \%$ if we use the dynamic values of sound speed and density (corresponding to the Hugoniot state behinda $50 \mathrm{kbar}$ shock). Again, this error is minuscule for the present discussion. It is interesting that, given experimental ejecta volume data, we can also invert (11) and infer a model-based value of the ablation pressure. These values are listed in the second column of Table 1 in parentheses. Such an inversion assumes that the ablated material makes a negligible contribution to the overall mass loss from the target for a single laser pulse. The very large model-inferred pressures suggest that there are strong limitations inherent in such a simple model. Remember that our model neglects surface ejecta, created by the ablative instability, and directly ablated material losses. Both of these contribute to the total mass loss from the target and, thus, the apparent driving pressure in the simple unfolding via Equations (11) and (12).

Within the basic model we can also estimate mean fragment sizes and velocities. The maximum radial velocity can be computed from (4):

$$
u(r)=\frac{2 P_{\max } \tau r}{\rho_{0} a^{2}},
$$

with the maximum value of $2 P_{\max } \tau /(\rho a)$ at $r=a$, once again assuming that $\rho=\rho_{0}$. We can use this velocity to infer nominal mean strain rates for the target, and thus mean fragment sizes in the context of the Grady-Kipp fragmentation theory [29]. The resulting estimates may be order of magnitude in accuracy for the fragment sizes. A strain rate can be computed from Equation (13):

$$
\dot{\varepsilon}=\frac{u_{\max }}{a}=\frac{2 P_{\max } \tau}{\rho_{0} a^{2}} .
$$


Table 1: Parameters and predictions for the cratering model. $\mathrm{P}_{\max }$ values labeled with * were inferred from the KrF VISAR experimental series. $P_{\max }$ The values of $P_{I}$ were found by inverting Equation (11). These values were calculated for a pulse with focal radius of 75 microns and a FWHM of $34 \mathrm{~ns}$. The experiments were discussed in [15].

\begin{tabular}{ccccccccc}
\hline \hline Material & $\begin{array}{c}\mathrm{P}_{\max } \\
(\mathrm{kbar})\end{array}$ & $\begin{array}{c}\mathrm{P}_{\mathrm{I}} \\
(\mathrm{kbar})\end{array}$ & $\begin{array}{c}\mathrm{\rho}_{0} \\
(\mathrm{~g} / \mathrm{cc})\end{array}$ & $\begin{array}{c}\mathrm{C}_{0} \\
(\mathrm{~km} / \mathrm{s})\end{array}$ & $\begin{array}{c}\mathrm{Y} \\
(\mathrm{kbar})\end{array}$ & $\begin{array}{c}\mathrm{V}_{\mathrm{c}} \\
\left(\mathrm{micron}^{3}\right)\end{array}$ & $\begin{array}{c}\mathrm{V}_{\mathrm{e}} \\
\left(\text { micron }^{3}\right)\end{array}$ & $\begin{array}{c}\mathrm{V}_{\operatorname{Exp}} \\
\left(\text { micron }^{3}\right)\end{array}$ \\
\hline $\mathrm{Sn}$ & 50.0 & 36 & 7.287 & 2.608 & 0.1 & $6.7 \times 10^{6}$ & $1.6 \times 10^{6}$ & $1.0 \times 10^{6}$ \\
$\mathrm{Fe}$ & 50.0 & 136 & 7.85 & 3.574 & 10.0 & $4.5 \times 10^{4}$ & $1.1 \times 10^{4}$ & $8.5 \times 10^{4}$ \\
$\mathrm{Cu}$ & $50.0^{*}$ & 100 & 8.93 & 3.94 & 3.0 & $1.2 \times 10^{5}$ & $2.9 \times 10^{4}$ & $1.2 \times 10^{5}$ \\
$\mathrm{Mo}$ & 50.0 & 228 & 10.206 & 5.124 & 16.0 & $1.5 \times 10^{4}$ & $3.7 \times 10^{3}$ & $8.0 \times 10^{4}$ \\
$\mathrm{Ta}$ & $50.0^{*}$ & 171 & 16.654 & 3.414 & 7.0 & $3.1 \times 10^{4}$ & $7.8 \times 10^{4}$ & $9.0 \times 10^{4}$ \\
$\mathrm{~W}$ & $60.0^{*}$ & 285 & 19.224 & 4.029 & 20.0 & $1.2 \times 10^{4}$ & $2.9 \times 10^{3}$ & $6.5 \times 10^{4}$ \\
$\mathrm{Au}$ & $60.0^{*}$ & 26 & 19.24 & 3.056 & 0.1 & $3.1 \times 10^{6}$ & $7.6 \times 10^{5}$ & $1.4 \times 10^{5}$ \\
\hline \hline
\end{tabular}

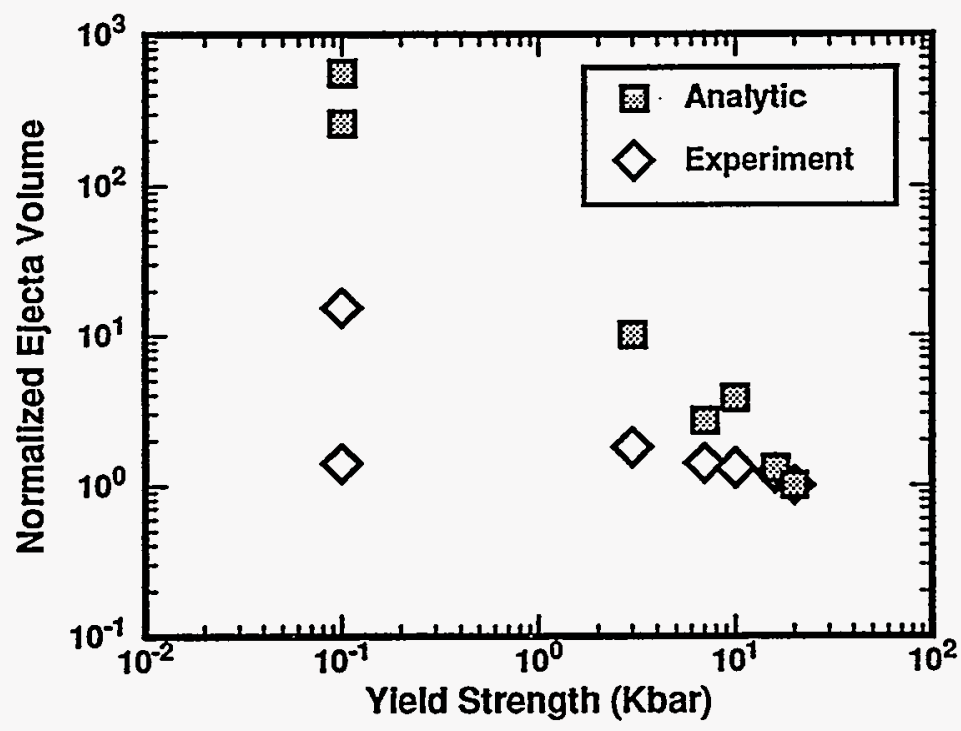

Fig. 12. Normalized crater volumes for experimental ejecta volumes and analytical model predictions as functions of target yield strength.

Applying this strain-rate formula to the theory discussed in [29] we arrive at the following formula for the mean fragment size (in units of length):

$$
S=\left(\frac{48 \gamma}{\rho_{0} \dot{\varepsilon}^{2}}\right)^{1 / 3}=\left(\frac{16 \gamma \rho_{0} a^{4}}{P_{\max }^{2} \tau^{2}}\right)^{1 / 3} .
$$

Equations (14) and (15) suggest that smaller faster fragments are produced as $P_{\text {max }}$ increases and as the laser pulsewidth increases. The fragments are also predicted to scale inversely in velocity and directly in size with the pulse focal radius, for constant $P_{\max }$. This is unexpected and it is not clear what its significance is. The constitutive parameter $\gamma$ is called the surface energy of the material, and is quite difficult to determine experimentally and theoretically for hot solids near the melt boundary, although it is bounded from below by the liquid surface tension which has been determined form many metals. In Table 2 we evaluate $u_{\max }$ and $S$ for targets for which we can approximate $\gamma$. These evaluations use the same nominal ablation pressures as are given in Table 1 . Remarkably, the sizes and velocities are well within the range of experimental data [14], although the velocities are at the high end of the observed distributions. These velocities are considerably larger than the material flow velocities observed in the CTH calculations after laser pulse tum-off. 


\section{Comments on Fragmentation in CTH Calculations}

The method of computing fragmentation in the CTH calculations uses the same formulas as the analytic model and its implementation is identical to that discussed in [29]. The only difference is that the dynamics of the fragmenting body in the present case is driven by a laser, rather than by explosives or impacts. The hydrocode also calculates a more precise evaluation of the space-time dependent strain rate through the cell-by-cell approach.

The fragmentation is not fully coupled to the material response in this approach. Rather, the material motion evolves without being influenced by the sub-grid scale fragmentation behavior. The fragment characteristics, as calculated by Equation (15), are applied in each computational cell through a passive calculation as time advances. This approach has been successfully tested for impact and explosive problems. It's success in the present case, of course, must be determined through comparison with data. A schematic of the passage from the analytic model to the detailed CTH calculation of fragmentation is presented in Figure 13.

Figure 13 also reveals one major issue that we do not really address in the present work. The experimental data available from the EUVL debris studies provides a lot of information about the distribution of fragment velocities as functions of ejection angle. In our calculations to this point we have not attempted to develop a reasonable statistical model of the angular distribution of the emitted debris. For the purposes of developing a testable angular distribution, we have simply assumed that the debris of size predicted by equation (15) travels with the same velocity vector as the parent material from which it was created. This is not unreasonable if the original material flow velocities are much larger than fracture velocity magnitudes determined from the strain rates. However, for EUVL problems the fracture velocities are of the same order as the material flow velocities, and therefore is not an accurate assumption. A better method must be determined, but this is a matter of some research, since far less is known about how to model statistical angular distributions from fragmenting bodies than how to model size distributions.

We have illustrated the kind of information that emerges from the coupled laser/hydrodynamics calculations in Figure 14. "Raw" CTH evaluation of the parent fragmented material is shown in Figure 14a. Each cell yields an individual set of fragment masses and sizes based on Equation (15). These are accumulated into overall fragment distribution data as a function of time. Total fragmented mass in the calculation quickly stabilizes to a constant value within approximately 1 microsecond of laser pulse turn-off. Thus, it makes sense to discuss a final distribution accumulated at the stopping time of the calculation. Such a distribution is presented in Figure 14b. There, we have plotted the fragment size versus binned mass distributions for two different laser pulse intensities in gold 2.0 microseconds after the pulse ends. A statistical procedure discussed in [29] was used to smooth the data.

A huge amount of information can be acquired from the CTH fragmentation simulations, including mass, size, velocity, ejection angles, momentum, kinetic energy, and thermodynamic state data. We expect that some of these data will not "stabilize" in time due to numerical noise and low amplitude ringing effects. We have not applied filtering techniques either to the raw data or to the choice of cells for performing fragmentation calculations. The possible problems that arise from this can be seen in Figure $14 \mathrm{~b}$, where we believe that there is too much predicted debris mass associated with very small fragment sizes for the illustrated cases as compared to experimental data. An appropriate filtering technique, either empirically or theoretically based, would eliminate this problem. We should also note that the Poisson statistical distribution that is used to describe the fragment size distribution contributes to excessive mass in the small fragment sizes, and may require modification.

We also find that our very simplistic treatment of the fragment velocities creates errors, for example in the angular distribution. For example, we have predicted for $\mathrm{KrF}$ pulses that the ejecta emerge at shallower angles from the target than are observed in experiments. One contributor to this problem is our overprediction of the ablation pressure from LASNEX for these pulses. But, another problem is that the parent material for much of the fragmented mass is moving at shallow angles. Because we arbitrarily define the fragment velocity to be that of the parent material (having no better theory at this point in time), we falsely encode this material motion on the ejecta. We have seen that fragment velocities can be larger than the magnitude of material velocities observed in CTH. Thus, a fragment could be strongly ejected normal to the parent material, and we would currently fail to predict it. 
Table 2: Calculated values of fragment velocity and fragment size for selected materials, using the same parameter values as in Table 1.

\begin{tabular}{ccccc}
\hline \hline Material & $\mathrm{P}_{\max }$ (kbars) & $\gamma\left(\mathrm{J} / \mathrm{m}^{2}\right)$ & $u_{\max }(\mathrm{m} / \mathrm{s})$ & $S$ (micron) \\
\hline $\mathrm{Sn}$ & 50.0 & 0.6 & $\sim 600$ & $\sim 4$ \\
$\mathrm{Fe}$ & 50.0 & 2.8 & $\sim 600$ & $\sim 7$ \\
$\mathrm{Cu}$ & 50.0 & 1.3 & $\sim 500$ & $\sim 6$ \\
$\mathrm{~W}$ & 60.0 & 2.8 & -300 & $\sim 9$ \\
\hline \hline
\end{tabular}
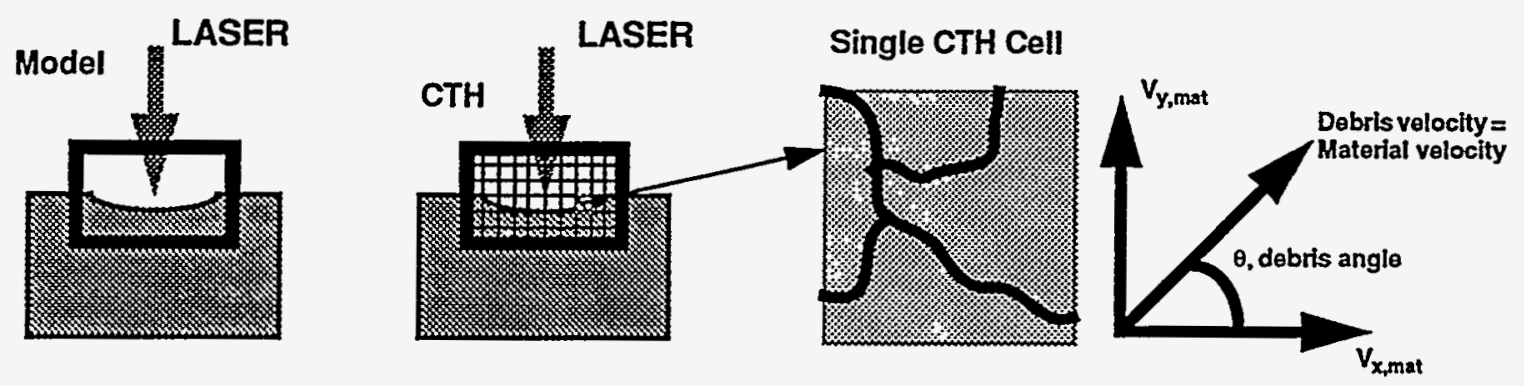

Fig. 13. Schematic of the passage from the analytic model fragment prediction to its application in a large-scale hydrodynamics code.
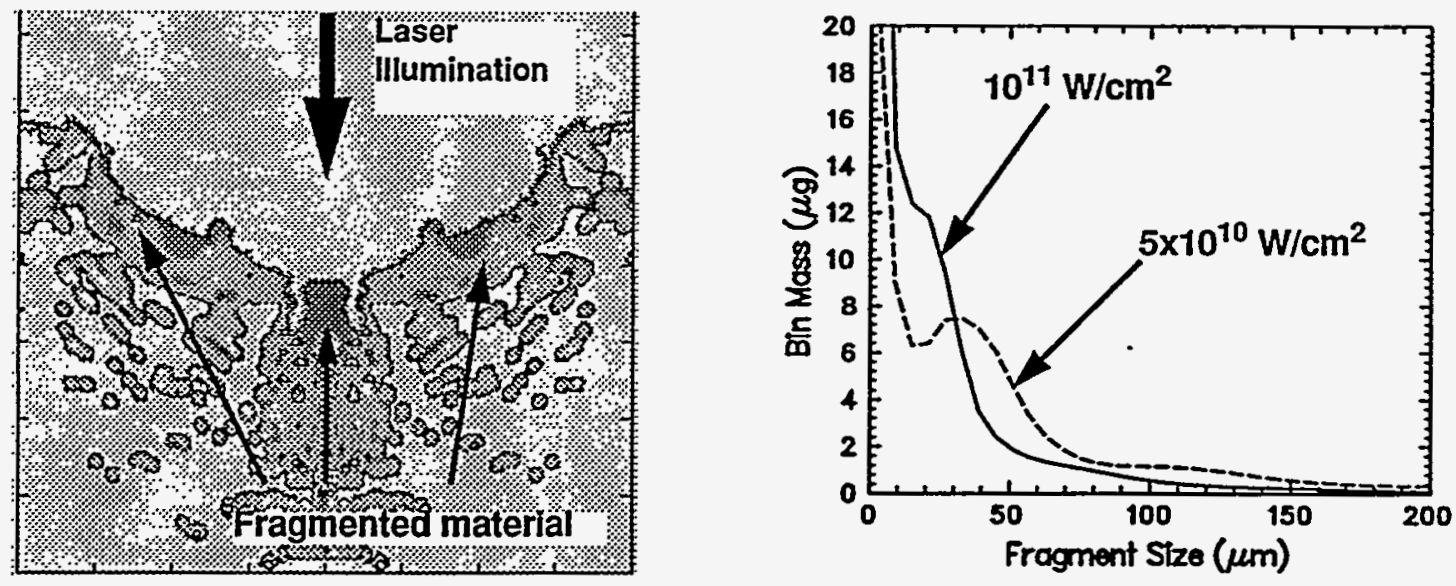

Fig. 14. Illustration of fragmentation calculations in CTH. The target is gold. For the first picture, the intensity is $1011 \mathrm{~W} / \mathrm{cm} 2$, and the time is 2 microseconds after the end of the laser pulse. For the second figure, the time is also 2 microseconds after the end of the laser pulse.

\section{Additional Remarks on the Ablation Pressure}

We now briefly retum to the question of determination of the ablation pressure that is so critical to driving the crater development and ejecta formation within a hypervelocity impact type analog of the EUVL debris process. The scaling of the ablation pressure with the material and laser parameters is an issue of great importance, historically rooted in ICF concerns. Many references discuss this scaling in the intensity range $I \geq 10^{13} \mathrm{~W} / \mathrm{cm}^{2}$. In the range of practical interest for this work, $10^{10}-10^{12} \mathrm{~W} / \mathrm{cm}^{2}$, there has been far less work, and the uncertainties in the actual scaling behavior are greater. The most general type of scaling relation has the form:

$$
P=F(A, Z, \tau, a, I, \lambda) .
$$




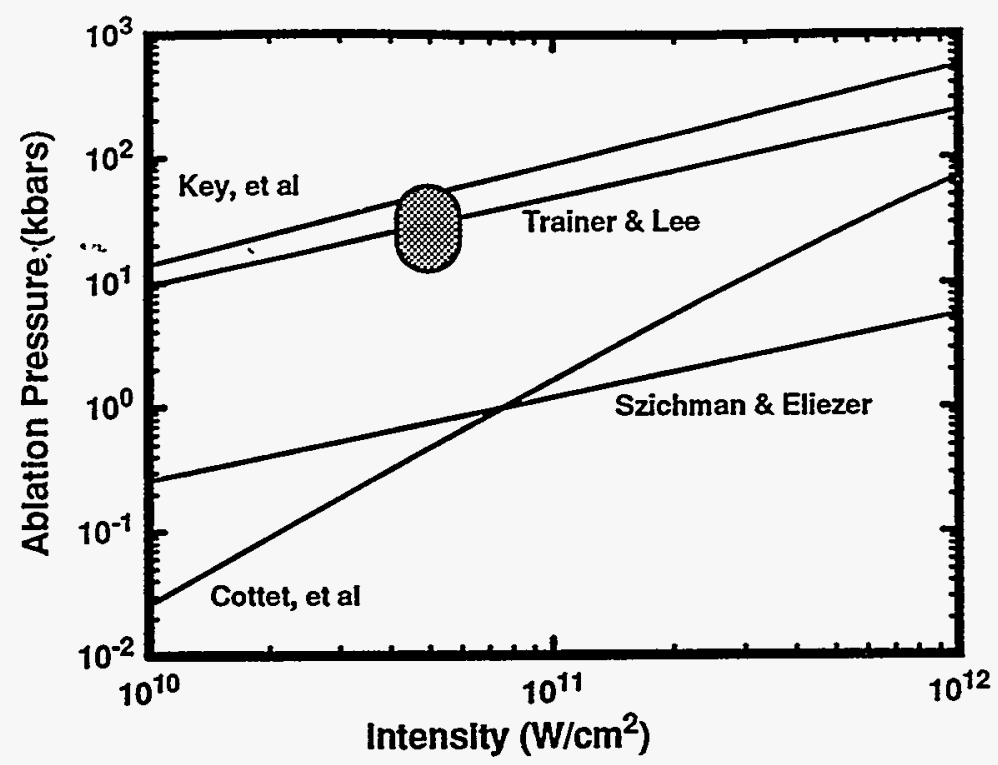

Fig. 15. Variation in scaling laws for ablation pressure versus laser intensity deduced from experiments and computer simulations. The shaded oval denotes a region where some of our KrF VISAR experiments were unfolded.

Typically, experimetits, analytic modeling, and computer calculations are used to determine a relation like Equation (16). For particular lasers and ranges of intensities Equation (16) can be simplified to be simply a * relationship between absorbed laser intensity and ablation pressure. For example, we have plotted five such relationships in the literature that at least neighbor on the intensity range that we are interested in Figure 15. The particular equations plotted in Figure 15 are written below.

Trainer and Lee [34] (material, wavelength independent):

$$
P=6.0\left(I / 10^{14}\right)^{0.7}
$$

Key, et al [35] (1064 nm laser light, material independent):

$$
P=3.5\left(I / 10^{13}\right)^{0.8}
$$

Cottet, et al [36]:

$$
P=0.12\left(I / 10^{14}\right)^{0.67}
$$

Szichman and Eliezer [37] (gold):

$$
P=8.14\left(I / 10^{14}\right)^{0.841} /\left(1+0.0147 \cdot I / 10^{14}\right)^{-0.992}
$$

These relations are rather typical of what is found in the literature. In particular, notice the wide disparity in predictions at low intensities and the fact that the experimental data is not accurately predicted by some of the relations. The relations tend to be more self consistent at higher intensities. This is reasonable, given the fact that these laws were determined from ICF related work.

\section{CONCLUSIONS}

We have acquired significant experimental, computational, and analytical understanding of the physics of condensed debris formation in laser-plasma sources for EUVL. To gain this understanding we have applied a wide variety of shock wave and impact physics concepts and computational tools that originated in the defense community. These tools are proving invaluable in understanding this important industrial technology.

The successes of our modeling efforts include a reasonably accurate picture of the dynamical evolu- 
tion of the debris, starting from the initial phases of the laser pulse absorption. We have also achieved at least qualitative agreement with unique experimental data. Some of this data, such as our time resolved measurements related to the ablation pressure induced in metal targets by lasers in the intensity range of $10^{10}-10^{12} \mathrm{~W} / \mathrm{cm}^{2}$, appear to be unique and important for validating both fragmentation models and laserplasma absorption physics in this non-ICF related intensity range.

Our computational modeling is fairly complex and is still being validated. There are technical aspects of the modeling that require improvements or new ideas in order to be more predictive. For example, we require some fundamentally new ideas in dynamic fragmentation in order to better predict the angular distribution of debris resulting from our laser pulse absorptions. In addition, the troublesome problems related to the physics of ablation of solid materials under laser light in the comparatively "cold" range require better understanding. These issues are not only important for EUVL, but would aid in the modeling of more general laser manufacturing processes and laser-tissue interactions.

When we started our work the levels of debris flux upon critical EUVL optical elements needed to be reduced by six orders of magnitude for commercial feasibility. Because of our careful campaign of experiment, modeling, and better engineering, at the current time this debris flux has been reduced in our experimental systems by three orders of magnitude. This is a major achievement. It is our hope that continued refinement of our knowledge will lead to enough reduction in current debris levels to make production-worthy EUVL with laser-plasma sources a reality.

\section{ACKNOWLEDGMENT}

This work performed at Sandia National Laboratories supported by the U. S. Department of Energy under contract number DE-AC04-94AL85000.

\section{REFERENCES}

1. Levenson, M. D. (1993), "Wavefront Engineering for Photolithography," Physics Today, July, 28-36.

2. Carruthers, J. A. (1993), "ULSI Lithography," in Soft X-Ray Projection Lithography, Volume 18, ed. by A. M. Hawryluk and R. H. Stulen, Optical Society of America, 2-5.

3. Keyes, R. W. (1993), "The Future of the Transistor," Scientific American, June, 70-78.

4. Atwood, D. (1992), "New Opportunities at Soft-X-Ray Wavelengths," Physics Today, August, 1992, 24-31.

5. Stulen, R. H. and R. R. Freeman.(1991), “Developing a Soft X-Ray Projection Lithography Tool," AT\&T Technical Journal, November/December, 37-48.

6. Berreman, D. N., et al (1990), "Soft X-Ray Projection Lithography Printing of $0.2 \mu \mathrm{m}$ Features using a 20:1 Reduction," Optics Lett., 15, 529-531.

7. Kubiak, G. D., et al (1991), "Diffraction-limited soft X-ray projection lithography with a laser plasma source," J. Vac. Sci. Technol. B, 9, 3184-3188.

8. Tichenor, D. A. (1991), "Diffraction-Limited Soft X-Ray Projection Imaging Using a Laser Plasma Source," Optics Lett., 16, 1557-1559.

9. Haney, S. J., K. W. Berger, G. D.Kubiak, P. D. Rockett, and J. Hunter (1993), "Prototype HighSpeed Tape Target Transport for a Laser Plasma Soft X-Ray Projection Lithography Source," Appl. Optics, 32, 6934.

10. Kubiak, G. D. et al (1994), "Characterization of an Expanded-Field Schwarzschild Objective for Extreme Ultraviolet Lithography," J. Vac. Sci. Technol. B, 12, 3820.

11. Alaterre, P., et al (1986), "X-Ray Conversion Efficiency as a Function of Atomic Number for 0.26 mm Laser-Irradiated Targets," Phys. Rev. A, 34, 4184-4194.

12. Chakir, M., et al (1988), "Laser Plasma X-Ray Sources for Microlithography," J. Appl. Phys., 63, 892-899.

13. Cerjan, C. (1993), "Spectral Characterization of Lithographic Sources," in Soft X-Ray Projection Lithography, Volume 18, ed. by A. M. Hawryluk and R. H. Stulen, Optical Society of America, 132137.

14. Silfvast, W. T., et al (1993), "Laser Plasma Source Characterization for SXPL," in Soft X-Ray Projection Lithography, Volume 18, ed. by A. M. Hawryluk and R. H. Stulen, Optical Society of America, 117-126. 
15. Kubiak, G. D., et al (1993), "Laser Plasma Source for SXPL: Production and Mitigation of Debris," in Soft X-Ray Projection Lithography, Volume 18, ed. by A. M. Hawryluk and R. H. Stulen, Optical Society of America, 127-131.

16. Early, K. and W. H. Arnold (1993), "Cost of Ownership for SXPL," in Soft X-Ray Projection Lithography, Volume 18, ed. by A. M. Hawryluk and R. H. Stulen, Optical Society of America, 14-22.

17. Kruer, W. L. (1988), The Physics of Laser Plasma Interactions, Addison-Wesley, Reading, MA.

18. Dingus, R. S. and B. P. Shafer (1990), "Laser-Induced Shock Wave Effects in Materials," SPIE 1202: Laser-Tissue Interactions, 36-45.

19. Zimmerman, G. and W. Kruer (1975), "Numerical Solution of Laser-Initiated Fusion," Comm. Plasma Phys., 2, 51-61.

20. Rockett, P. D., et al (1991), "XUV Conversion Efficiency in a Low Intensity KrF Laser-Plasma for Projection Lithography," in Soft X-Ray Projection Lithography, Volume 16, Optical Society of America.

21. Rockett, P. D. et al (1993), "The Investigation of Discharge-laser-driven Plasmas as Sources for Soft-X-Ray Projection Lithography", in Soft X-Ray Projection Lithography, Volume 18, ed. by A. M. Hawryluk and R. H. Stulen, Optical Society of America, 163-169.

22. Olson, R. E., W. C. Sweatt, and P. D. Rockett (1993), "Computational Simulations of a Soft X-Ray Projection Lithography Laser Plasma Source," in Soft X-Ray Projection Lithography Technical Digest, 118-120.

23. McGlaun, J. M., S. L. Thompson, and M. G. Elrick (1990), "CTH: A Three-Dimensional Shock Wave Physics Code," Int. J. Impact Engng., 10, 351-360.

24. Pirri, A. N. (1977), "Theory for Laser Simulation of Hypervelocity Impact," Phys. Fluids, 20, 221228.

25. Nebelsine, P. E. (1976), "Laser Simulation of Hypervelocity Impact," in AIAA 14th Aerospace Sciences Meeting Proceedings, AIAA Paper No. 76-52.

26. Vorob'ev, V. S. (1993), "Plasma arising during the interaction of laser radiation with solids," Physics - Uspekhi, 36, 1129-1157.

27. Silfvast, W. T., et al (1991), "Laser Plasma Source Characterization for SXPL," in Soft X-Ray Projection Lithography, Volume 18, ed. by A. M. Hawryluk and R. H. Stulen, Optical Society of America, 117-131.

28. Bender, H. A., D. O'Connell, and W. T. Silfvast, (1995), "Velocity Characterization of Particulate Debris from Laser-produced Plasmas used for Extreme-Ultraviolet Lithography", Appl. Optics, submitted.

29. Kipp, M. E., D. E. Grady, and J. W. Swegle (1993), "Numerical and Experimental Studies of HighVelocity Impact Fragmentation," Int. J. Impact Engng., 14, 427-438.

30. Chhabildas, L. C. (1987), "Survey of Diagnostic Tools Used in Hypervelocity Impact Studies," Int. J. Impact Engng., 5, 205-220.

31. Nebolsine, P. E. (1976), "Laser Simulation of Hypervelocity Impact," in AIAA 14th Aerospace Science Meeting, Washington, D.C., AIAA Paper No. 76-52.

32. Trucano, T. G., et al (1993), "Computational Analysis of Debris Formation in SXPL Laser-Plasma Sources," in Soft X-Ray Projection Lithography, Volume 18, ed. by A. M. Hawryluk and R. H. Stulen, Optical Society of America, 146-150.

33. Melosh, H. J. (1989), Impact Cratering, a Geologic Process, Oxford U. Press, New York.

34. Trainer, R. J. and Y. T. Lee (1982), "Analytic Models of Laser-Generated Shock-Wave Experiments," Phys. Fluids, 25, 1898-1907.

35. Key, M. H. et al (1983), "A Study of Ablation by Laser Irradiation of Plane Targets at Wavelengths $1.05,0.53$, and $0.35 \mu \mathrm{m}$," Phys. Fluids, 26, 2011-2026.

36. Cottet, F. et al (1984), "Measurement of Laser Driven Shock Pressures and Estimate of Energy Lost at $1.05 \mu \mathrm{m}$ Wavelength," J. Appl. Phys., 55, 4125-4127.

37. Szichman, H. and S. Eliezer (1992), "Scaling Laws for Pressure, Temperature, and Ionization With Two-Temperature Equations-of-State Effects in Laser Produced Plasmas," Lasers Part. Beams, 10, $23-40$. 